\title{
Bismarck, die Konservativen und der Kulturkampf im Deutschen Reich
}

\section{Bismarck, the Conservatives and „Kulturkampf" in German Empire}

Hans-Christof Kraus / Hans-Christof.Kraus@uni-passau.de

Lehrstuhl für neuere und neueste Geschichte, Philosophische Fakultät, Universität Passau, DE

\begin{abstract}
The text deals with a complicated period in German history, namely the creation of a unified state in the 19th century. It discusses the relationship of the church and the state from 1815 till the 1980s and focuses on Bismarck's "Kulturkampf" that flared up after the beginning of German empire. The author presents Birmarck's intentions and the positions of other participants, first of all Catholic and Protestant churches.
\end{abstract}

\section{Keywords}

national state, Germany, Otto von Bismarck, "Zentrum" Party, conservatives, "Kulturkampf" (Culture wars) 
Die Auseinandersetzung zwischen Staat und Kirche ${ }^{1}$, auch zwischen der aufsteigenden bürgerlich-liberalen Bewegung einerseits und den traditionellen religiösen Institutionen und Lebensformen andererseits gehört zu den Grundkonstanten der Geschichte des neunzehnten Jahrhunderts, durchaus nicht nur in Deutschland ${ }^{2}$. Aber in Deutschland gab es - und gibt es bis heute - eine Besonderheit, die diesen Konflikt im Vergleich zu den meisten anderen Ländern bedeutend verschärft hat und auch verschärfen musste: Gemeint ist die Tatsache der seit der Reformation bestehenden deutschen Bikonfessionalität, also das Faktum eines in etwa gleich starken Anteils von Katholiken und Protestanten in der Bevölkerung. Dieses Faktum hat dazu geführt, dass schon seit mehreren Jahrhunderten immer wieder nach Möglichkeiten für einen doppelten Ausgleich gesucht werden musste, um zum einen das Verhältnis zwischen den beiden großen Konfessionen in Deutschland auf eine für beide Seiten akzeptable und allgemein verträgliche, vor allem aber friedliche Weise zu regeln, - und um zum anderen die Beziehungen zwischen Staat und Kirche immer wieder neu auszutarieren; das Ziel bestand in beiden Fällen darin, zu einer nach Möglichkeit konfliktfreien Formierung des beiderseitigen Verhältnisses zu gelangen³.

Diese Regelung der Beziehung zwischen Kirche und Staat gestaltete sich seit Beginn des neunzehnten Jahrhunderts allerdings zunehmend schwieriger, und dies hatte wiederum mehrfache Ursachen, von denen nur die beiden wichtigsten genannt sein sollen: erstens die neue territoriale Gliederung Deutschlands durch den Wiener Kongress von 1815, der die althergebrachte konfessionelle Homogenität der allermeisten deutschen Staaten und Länder weitgehend beseitigte, dafür aber eine Vielzahl religiös-konfessionell gemischter Einzelstaaten im Deutschen Bund schuf $f^{4}$; und zweitens eine deutliche Ausdehnung der Staatstätigkeit auf alle Bereiche nicht nur der Politik im Allgemeinen, sondern auch des sozialen und gesellschaftlichen Lebens im Besonderen. Vor allem die in dieser Zeit beginnende ökonomisch-technische Modernisierung erforderte eine bedeutende Vermehrung der Verwaltungstätigkeit des Staates und damit auch eine Verstärkung des staatlichen Anspruchs auf die Regelung der in Bewegung geratenen gesellschaftlich-sozialen Verhältnisse ${ }^{5}$.

Man wird im Rückblick sagen müssen, dass es erst gegen Ende des 19. Jahrhunderts in Deutschland gelungen ist, das Verhältnis zwischen Staat und Kirche auf eine für beide Seiten einigermaßen zufriedenstellende und akzeptable Weise neu zu regeln und

1 Der Text entspricht im Wesentlichen meinem Vortrag, den ich am 24. April 2013 an der Tomáš-Masaryk-Universität in Brünn / Masarykova univerzita Brno gehalten habe.

2 Gut zusammenfassender Überblick bei Rémond, René: Religion und Gesellschaft in Europa. Von 1789 bis zur Gegenwart. München 2000.

3 Einen zuverlässigen, materialreichen und reflektiert argumentierenden Überblick hierzu liefert Nowak, Kurt: Geschichte des Christentums in Deutschland. Religion, Politik und Gesellschaft vom Ende der Aufklärung bis zur Mitte des 20. Jahrhunderts. München 1995; anregend ebenfalls Friedrich, Martin: Kirche im gesellschaftlichen Umbruch. Das 19. Jahrhundert (Zugänge zur Kirchengeschichte 8). Göttingen 2006.

4 Vgl. Nipperdey, Thomas: Deutsche Geschichte 1800-1866 - Bürgerwelt und starker Staat. München 1983, S. $403 \mathrm{ff}$.

5 Zusammenfassende Darstellung durch verschiedene Verfasser in: Deutsche Verwaltungsgeschichte. Hrsg. v. K. G. A. Jeserich; H. Pohl; G.-Ch. von Unruh. Bd. 2: Vom Reichsdeputationshauptschluß bis zur Auflösung des Deutschen Bundes. Stuttgart 1983. 
auszutarieren $^{6}$. In der Zwischenzeit aber gab es eine Reihe von teilweise schweren und belastenden Konflikten, die man in Deutschland seither als „Kulturkämpfe“ bezeichnet ${ }^{7}$. Als der zweifellos schwerste und folgenreichste dieser Kulturkämpfe muss die Auseinandersetzung in Preußen und im Deutschen Reich angesehen werden, die sich in den 1870er Jahren unter der Regierung Bismarcks abspielte und zu Anfang der 1880er Jahre nur mühsam zum Abschluss gebracht werden konnte ${ }^{8}$.

Von dieser Auseinandersetzung wird also im Folgenden die Rede sein; im ersten Teil soll die Entwicklung des Verhältnisses zwischen Staat und Kirche in Deutschland seit der Säkularisation des Jahres 1803 skizziert werden, bevor im zweiten Teil der äußere Verlauf des von Bismarck ausgelösten Kulturkampfs im neugegründeten Deutschen Kaiserreich $\mathrm{zu}$ behandeln ist und dabei auch die eigentlichen politischen Motive des ersten deutschen Reichskanzlers in diesem Zusammenhang beleuchtet werden sollen. Der dritte Teil wird den Widerständen gegen die staatlichen Kulturkampfmaßnahmen gewidmet sein, während im abschließenden vierten Teil die Ergebnisse und Folgen des Ende der 1870er Jahre abgebrochenen Konflikts knapp thematisiert werden sollen.

\section{I.}

Obwohl am Beginn der deutschen Kirchengeschichte des 19. Jahrhunderts ein bedeutender Vorgang der Säkularisierung steht, wird man nicht behaupten können, dass Kirche, Religion und Glaube als solche hierdurch überhaupt an den Rand geschoben oder in die Bedeutungslosigkeit abgedrängt worden seien. Der Historiker Thomas Nipperdey hat es einmal sehr treffend folgendermaßen formuliert: „Das 19. Jahrhundert ist bewegt vom Kampf um Christentum und Modernität, aber gerade darin behauptet sich das Christentum, formt sich um, erneuert sich, ja gewinnt ganz außerordentlich an öffentlicher Geltung und an Durchsetzungskraft. Die Religion wie die Kirchen ragen nicht als ein Relikt der Tradition in das 19. Jahrhundert hinein, sondern sie sind Produkte und gestaltende Mächte dieses Jahrhunderts zugleich“9.

6 Vgl. Friedrich, M.: Kirche im gesellschaftlichen Umbruch, S. 123 ff.

7 Einen älteren, aber immer noch brauchbaren Überblick liefert: Franz, Georg: Kulturkampf. Staat und katholische Kirche in Mitteleuropa, München 1954.

8 Die Deutung und Darstellung dieses Konflikts wird bis heute durch zumeist einseitige Sichtweisen und Wertungen bestimmt; das gilt für die z. T. sehr detailreichen, immer noch wichtigen Darstellungen von Kißling, Johannes B.: Geschichte des Kulturkampfes im Deutschen Reiche. Bde. 1-3. Freiburg i. Br. 1911-1916; Schmidt-Volkmar, Erich: Der Kulturkampf in Deutschland 1871-1890. Göttingen - Berlin - Frankfurt/M. 1962; Lill, Rudolf: Der Kulturkampf in Preußen und im Deutschen Reich. In: Handbuch der Kirchengeschichte. Hrsg. v. H. Jedin. Bd. VI/2. Freiburg in Br. - Basel - Wien 1973, S. 28-48; Morsey, Rudolf: Der Kulturkampf. In: Der soziale und politische Katholizismus. Entwicklungslinien in Deutschland 1803-1963. Hrsg. v. A. Rauscher, Bd. I, München 1981, S. 72-109; Nipperdey, Thomas: Deutsche Geschichte 1866-1918. Bd. 2: Machtstaat vor der Demokratie. München 1992, S. 364-381 als fundierte Überblicksdarstellung bleibt grundlegend: Huber, Ernst R.: Deutsche Verfassungsgeschichte seit 1789. Bd. IV. Stuttgart - Berlin - Köln Mainz $1982^{2}$, S. 645-831.

9 Nipperdey, T.: Deutsche Geschichte 1800-1866, S. 403; vgl. auch Rémond, R.: Religion und Gesellschaft in Europa, S. $31 \mathrm{ff}$. 
Die Religion blieb also weiterhin eine gestaltende Lebensmacht, die einen entscheidenden Einfluss auf das soziale und gesellschaftliche Leben beider Konfessionen in Deutschland ausübte. Man wird sogar sagen können, dass die Revolutions-, Kriegs- und Umbruchszeit der Epoche zwischen 1792 und 1815 am Ende zur Entstehung einer neuen Religiosität, einer verstärkten Frömmigkeit innerhalb breiter Volksschichten geführt hat ${ }^{10}$; viele Menschen versuchten auf diese Weise, die negativen Erfahrungen der vorangegangenen Zeit zu bewältigen und neue geistige Orientierung zu finden. Zur Grundtendenz der Rechristianisierung nach 1815 gehörten nicht nur die Entstehung eines neuen christlichen Presse- und Publikationswesens, sondern auch die Herausbildung neuer „Frömmigkeitswelten“"11, gleichzeitig ebenfalls Neugründungen von Klöstern sowie die Zunahme von Wallfahrten im katholischen Bereich, - sondern andererseits auch die Erneuerung bestimmter Formen pietistischer Frömmigkeit im Protestantismus, die man als Erweckungsbewegung bezeichnete ${ }^{12}$.

Das betraf allerdings nicht die politische und institutionelle Stellung vor allem der katholischen Kirche, die in früherer Zeit, also bis zum Beginn des 19. Jahrhunderts, im alten Deutschen Reich eine außerordentlich bedeutende Position eingenommen hatte. Drei der Kurfürsten waren Erzbischöfe gewesen, die zugleich als Regenten bedeutender deutscher Territorien, vor allem im Westen Deutschlands, politische Macht ausgeübt hatten. Daneben hatte es ebenfalls noch weitere, allerdings kleinere geistliche Staaten im alten Deutschen Reich gegeben ${ }^{13}$. Mit dem Reichsdeputationshauptschluss von 1803 hörten die geistlichen Fürstentümer Deutschlands allerdings zu bestehen auf; sie wurden aufgeteilt und - unter französischem Druck - weltlichen Territorien zugeschlagen. Parallel zur großen politisch-sozialen Reformbewegung in Deutschland, vor allem in Preußen, Bayern und den mittleren und kleineren Rheinbundstaaten, wurden eine Fülle von kirchlichen Institutionen, darunter nicht nur Klöster, sondern auch Universitäten und Schulen, aufgelöst ${ }^{14}$.

Alle diese Vorgänge hatten bedeutende Folgen für die weitere Entwicklung des Verhältnisses zwischen Staat und Kirche (in diesem Fall besonders der katholischen Kirche)

10 Siehe dazu und zum Folgenden neben der klassischen Abhandlung von Holl, Karl: Die Bedeutung der großen Kriege für das religiöse und kirchliche Leben innerhalb des Protestantismus. In: Derselbe: Gesammelte Aufsätze zur Kirchengeschichte. Bd. 3. Tübingen 1928, S. 302-384, hier S. 347 ff., auch Schnabel, Franz: Deutsche Geschichte im neunzehnten Jahrhundert. Bd. 4. Die religiösen Kräfte. Freiburg i. Br. 1937, bes. S. 44 ff., $297 \mathrm{ff}$.

11 Dazu eingehend Nowak, K.: Geschichte des Christentums in Deutschland, S. 94 ff.; Friedrich, M.: Kirche im gesellschaftlichen Umbruch, S. $149 \mathrm{ff}$.

12 Vgl. Kantzenbach, Friedrich W.: Die Erweckungsbewegung. Studien zur Geschichte ihrer Entstehung und ersten Ausbreitung in Deutschland. Neuendettelsau 1957; Beyreuther, Erich: Die Erweckungsbewegung. In: Die Kirche in ihrer Geschichte. Hrsg. von K. D. Schmidt; E. Wolf. Bd. IV. Lieferung R (1. Teil). Göttingen 1963.

13 Vgl. Hartung, Fritz: Deutsche Verfassungsgeschichte vom 15. Jahrhundert bis zur Gegenwart. Stuttgart 1964', S. 142-147; allgemein auch den zusammenfassenden Überblick bei Boldt, Hans: Deutsche Verfassungsgeschichte. Bde. 1-2. München 1984-1990, hier Bd. 1, S. 262 ff.

14 Vgl. Boldt, H.: Deutsche Verfassungsgeschichte, Bd. 1, S. 283 f.; Nowak, K.: Geschichte des Christentums in Deutschland, S. 44 ff.; Lill, Rudolf: Reichskirche - Säkularisation - Katholische Bewegung. In: Der soziale und politische Katholizismus. Entwicklungslinien in Deutschland 1803-1963. Hrsg. v. Anton Rauscher. Bd. 1, München 1981, S. 15-45. 
in Deutschland, denn auf der einen Seite war die politische, verfassungsrechtliche und institutionelle Stellung der Kirche durch die schweren materiellen wie auch ideellen Verluste nach 1803 - und noch einmal nach 1815 - schwer beeinträchtigt worden. Auf der anderen Seite aber erhöhten die neuen Frömmigkeits- und Rechristianisierungstendenzen dieser Zeit ebenso die Stellung der Kirche im Rahmen des neu entstehenden und aufstrebenden religiösen Lebens in Deutschland nach den Befreiungskriegen. Freilich war und blieb das Verhältnis zwischen Staat und Kirche immer noch umstritten und prekär ${ }^{15}$ - nicht zuletzt, da man es im Jahr 1815 vermieden hatte, dieses Problem für ganz Deutschland im Rahmen des neuen Bundesrechts zu regeln, denn ein spezielles „Bundeskirchenrecht“ gab es jetzt und auch später nicht ${ }^{16}$. Und das bedeutete: Jeder einzelne Mitgliedsstaat des Deutschen Bundes musste versuchen, dieses Verhältnis in eigener Regie und vor dem Hintergrund der jeweils gegebenen regionalen Besonderheiten und Traditionen auf neue Grundlagen zu stellen.

Hieraus entstand nun eine Fülle von Problemen, die wiederum vor allem die katholische Kirche betrafen. Nur drei einzelne Aspekte seien hier besonders erwähnt:

1. die Frage der Ernennung neuer Bischöfe und Erzbischöfe; in dieser Angelegenheit beanspruchte der Staat seit 1815 ein deutliches Mitsprache- und Mitentscheidungsrecht, das gelegentlich durchaus einer massiven Einflussnahme auf die Besetzung hoher kirchlicher Ämter gleichkam ${ }^{17}$. Die von der Kirche bevorzugten streng rom- und papsttreuen Kandidaten wurden oftmals seitens der politischen Führungen der betroffenen Länder abgelehnt; hier bevorzugte man im allgemeinen eher gemäßigt orientierte, weniger streng auf die kirchliche Hierarchie ausgerichtete Geistliche, die auch eher bereit waren, staatlichen Ansprüchen im geistlich-kirchlichen Bereich nachzukommen und Eingriffe der Politik zu akzeptieren.

2. ist zu nennen die Frage der geistlichen Schulaufsicht und das Problem der kirchlichen Einflussnahme auf die Besetzung der Lehrstühle an den katholisch-theologischen Fakultäten der deutschen Universitäten sowie überhaupt der immer noch keineswegs unbedeutende kirchliche Einfluss auf das bestehende Bildungswesen und dessen Institutionen - auch dies blieb seit 1815 ein ständiger Konfliktherd mit konträren wechselseitigen Ansprüchen ${ }^{18}$; und

3. muss in diesem Zusammenhang die Frage des Eherechts und das Problem der interkonfessionellen christlichen Ehen erwähnt werden ${ }^{19}$. Infolge der Neuordnung

15 Allgemein hierzu: Huber, Ernst R.: Deutsche Verfassungsgeschichte seit 1789. Bde. 1-8. Stuttgart - Berlin - Köln - Mainz 1975-1990, hier Bd. 1, S. 387 ff., 416 ff.; Hollerbach, Alexander: Katholische Kirche und Katholizismus vor dem Problem der Verfassungsstaatlichkeit. In: Der soziale und politische Katholizismus. Entwicklungslinien in Deutschland 1803-1963. Hrsg. v. A. Rauscher. Bd. I. München 1981, S. 46-71.

16 Vgl. Huber, E. R.: Deutsche Verfassungsgeschichte, Bd. 1, S. 415 f.

17 Vgl. Nipperdey, T.: Deutsche Geschichte 1800-1866, S. 416 f.

18 Vgl. Jeismann, Karl-Ernst: Zur Bedeutung der „Bildung“ im 19. Jahrhundert. In: Handbuch der deutschen Bildungsgeschichte. Bd. 3: 1800-1870. Von der Neuordnung Deutschlands bis zur Gründung des Deutschen Reiches. Hrsg. v. K.-E. Jeismann; P. Lundgreen. München 1987, S. 1-21, hier S. 14 ff.

19 Vgl. als Überblick: Coing, Helmut: Die Auseinandersetzung um kirchliches und staatliches Eherecht im Deutschland des 19. Jahrhunderts. In: Christentum und modernes Recht. Beiträge zum Problem der Säkularisierung. Hrsg. v. G. Dilcher; I. Staff. Frankfurt a. M. 1984, S. 360-375. 
Deutschlands waren in vielen deutschen Bundesstaaten gemischt-konfessionelle Territorien entstanden, so dass hier in zunehmendem Maße das Problem gemischt konfessioneller (evangelisch-katholischer) Ehen auftauchte. Solche Eheschließungen wurden zwar akzeptiert, doch an der Frage der jeweiligen religiösen Erziehung der Kinder und deren Konfessionszugehörigkeit entzündete sich bald ein heftiger Streit.

Dies traf vor allem auf Preußen zu, das seit dem Wiener Kongress über bedeutende, zum großen Teil mehrheitlich katholische Westprovinzen im Rheinland und in Westfalen verfügte. Hier kam es in den Jahren 1837 bis 1840 zu einer heftigen konfessionspolitischen Auseinandersetzung, da die Kurie in Rom bestimmte informelle Absprachen nicht mehr anerkannte, die der preußische Staat mit einem verstorbenen Erzbischof von Köln getroffen hatte. Dieser Konflikt ${ }^{20}$ weitete sich zu einer Krise aus, auf deren Höhepunkt der neue Erzbischof von Köln von der Berliner Regierung abgesetzt und kurze Zeit inhaftiert wurde. Erst dem im Jahr 1840 auf den Thron gelangten neuen preuBischen König Friedrich Wilhelm IV. gelang eine Beilegung des Streits, indem er den Forderungen der Kirche weit entgegenkam, um die Auseinandersetzung, die inzwischen großes Aufsehen erregt und beträchtlichen politischen Schaden angerichtet hatte, so rasch wie möglich zu beenden ${ }^{21}$.

Der deutsche Protestantismus besaß im 19. Jahrhundert eine wesentlich staatsnähere innere Verfassung als der Katholizismus; seit jeher existierten evangelische Landeskirchen, die schon seit Luthers Zeiten eng verbunden waren mit den jeweiligen deutschen Territorialfürsten, den Landesherren; diese stellten zugleich innerhalb ihrer Territorien das weltliche Oberhaupt jeder dieser Landeskirchen dar, eben hierin bestand der Kern des deutschen landesherrlichen Kirchenregiments. Aber auch im evangelischen Deutschland gab es zeitweilig schwere Konflikte - vor allem um die seit 1817 vom preuBischen König und einigen anderen deutschen Fürsten verfügte protestantische Union zwischen Lutheranern und Reformierten ${ }^{22}$. Diese Union wurde keineswegs von allen der hiervon betroffenen evangelischen Christen beider früherer Konfessionen akzeptiert, und es sollte Jahrzehnte dauern, bis die teilweise schweren Auseinandersetzungen hierüber beendet werden konnten; am Ende setzte sich der Staat durch ${ }^{23}$.

Andere Auseinandersetzungen entstanden um die Reform der inneren protestantischen Kirchenverfassung: Hier drehte sich der Streit um die Frage der Aufrechterhaltung des

20 Hierzu siehe Schrörs, Heinrich: Die Kölner Wirren (1837) - Studien zu ihrer Geschichte. Berlin-Bonn 1927; vgl. ebenfalls Schnabel, F.: Deutsche Geschichte im neunzehnten Jahrhundert. Bd. 4, S. 106-164; zusammenfassende Darstellungen: Huber, E. R.: Deutsche Verfassungsgeschichte seit 1789, Bd. 2, S. 226-268; Hürten, Heinz: Kurze Geschichte des deutschen Katholizismus 1800-1960. Mainz 1986, S. 62-78, 274.

21 Vgl. Bußmann, Walter: Zwischen Preußen und Deutschland. Friedrich Wilhelm IV. Berlin 1990, S. 169 ff.

22 Vgl. Nipperdey, T.: Deutsche Geschichte 1800-1866, S. 432 f.; Friedrich, Kirche im gesellschaftlichen Umbruch (wie Anm. 3), S. 57 ff.

23 Siehe hierzu u. a. Friedrich, M.: Kirche im gesellschaftlichen Umbruch, S. 72 ff.; Thadden, Rudolf von: Die Geschichte der Kirchen und Konfessionen. In: Handbuch der preußischen Geschichte. Bd. 3. Hrsg. v. Wolfgang Neugebauer. Berlin - New York 2001, S. 547-711, hier S. 581 ff.; aus der älteren Forschung vor allem Foerster, Erich: Die Entstehung der Preußischen Landeskirche unter der Regierung Friedrich Wilhelms des Dritten nach den Quellen erzählt. Ein Beitrag zur Geschichte der Kirchenbildung im deutschen Protestantismus. Bd. 2. Tübingen 1907, S. 251 ff. 
staatlich bestimmten, monarchisch geprägten Kirchenregiments oder um die Einführung einer begrenzten Mitbestimmung der Gemeindeangehörigen innerhalb der Kirche durch Synoden, die man als Kirchenparlamente im eigentlichen Sinne bezeichnen kann. Auch dieser Konflikt innerhalb des deutschen Protestantismus, in den bereits der politische Gegensatz zwischen Konservatismus und Liberalismus stark hineinspielte, hat sich bis zur Reichsgründung von 1871, partiell noch bis zum Ende des Kaiserreichs hingezogen, wenngleich es in einigen Ländern zur Einführung synodaler Institutionen kam²4.

Kurz gesagt: Das Verhältnis zwischen Staat und Kirche war und blieb im Falle beider Konfessionen sehr konfliktreich und kontrovers; als wichtiger Höhepunkt vor dem folgenreichen Kulturkampf der 1870er Jahre kann die im August 1848 in der deutschen Nationalversammlung in Frankfurt am Main geführte, heftige und kontroverse Kirchendebatte angesehen werden, die vor allem der Frage des künftigen Verhältnisses zwischen Staat und Kirche in Deutschland gewidmet war. Diese Debatte hatte jedoch angesichts des weitgehenden Scheiterns der Ziele der Revolution - wenigstens vorerst - keine konkreten Folgen ${ }^{25}$.

\section{II.}

Der 1872 im Deutschen Reich, vor allem in Preußen und in Bayern, ausbrechende Konflikt zwischen Staat und Kirche wurde und wird seit 1873 mit dem Begriff „Kulturkampf“ bezeichnet; er geht zurück auf eine Äußerung des radikal-liberalen deutschen Mediziners und Parlamentsabgeordneten Rudolf Virchow ${ }^{26}$, der im preußischen Landtag die Deutsche Fortschrittspartei vertrat. Das Ziel seiner Partei und der von ihr angestrebten Politik bestehe darin, äußerte Virchow am 17. Januar 1873, „die Freiheit der individuellen, religiösen Überzeugung oder des religiösen Glaubens“ herzustellen - und hierzu bedürfe es wiederum, so drückte er sich aus, der „Emanzipation des Staates“, also einer wirklichen „Verweltlichung des Staates in allen seinen Richtungen“. Zur modernen Kultur als solcher könne die traditionsorientierte, damit rückwärtsgewandte römische Kirche, in der die Hierarchie inzwischen zum Selbstzweck geworden sei, nichts mehr beitragen, und aus diesem Grund handele es sich beim Kampf zwischen den Anhängern der alten Kirche und des neuen Fortschritts um nichts weniger als „um einen großen Kulturkampf“27.

24 Vgl. die ausführliche Darstellung von Besier, Gerhard: Preußische Kirchenpolitik in der Bismarckära. (Veröffentlichungen der Historischen Kommission zu Berlin 49), Berlin - New York 1980; sowie allgemein Nipperdey, T.: Deutsche Geschichte 1800-1866, S. 433 ff.; Friedrich, M.: Kirche im gesellschaftlichen Umbruch, S. $78 \mathrm{ff}$.

25 Die Texte In: Stenographischer Bericht über die Verhandlungen der deutschen constituirenden Nationalversammlung zu Frankfurt am Main. Hg. F. Wigard. Bd. 3, Frankfurt a. M. 1848, S. 1623-1799. Hierzu vgl. neben Becker, Winfried: Bürgerliche Freiheit und Freiheit der Kirche im Epochenjahr 1848. In: Jahres- und Tagungsbericht der Görres-Gesellschaft 1998. Köln o. J. (1999), S. 47-69, bes. 52-65. Auch Delius, Walter: Die Evangelische Kirche und die Revolution 1848. (Kirche in dieser Zeit 6/7). Berlin 1948, S. 43 ff., sowie Nowak, K.: Geschichte des Christentums in Deutschland, S. 122 ff., und Friedrich, M.: Kirche im gesellschaftlichen Umbruch, S. $97 \mathrm{ff}$.

26 Vgl. Goschler, Constantin: Rudolf Virchow. Mediziner - Anthropologe - Politiker. Köln 2002.

27 Siehe die Nachweise in: Franz, G.: Kulturkampf, S. 9 f., 295, und die Bemerkungen zum Begriff bei Huber, E. R.: Deutsche Verfassungsgeschichte, Bd. 4, S. $642 \mathrm{f}$. 
Damit war mit einem Mal das Stichwort geprägt, mit dem diese große Kontroverse und später auch vergleichbare Auseinandersetzungen bezeichnet werden sollten. Es handelte sich, wenn man auf die Ursachen des deutschen Konflikts der 1870er Jahre blickt, aber nicht nur um einen kulturell, religiös und konfessionell motivierten Zusammenstoß zwischen den Anhängern einer christlich und einer säkular geprägten Kultur, sondern vor allem auch - und dies in sehr starkem Maße - um eine genuin politisch motivierte Auseinandersetzung. Denn hier kamen viele unterschiedliche Voraussetzungen zusammen, so etwa der Streit um das 1870 verkündete Unfehlbarkeitsdogma des Ersten Vatikanischen Konzils, sodann ebenfalls die Auseinandersetzung um den von Papst Pius IX. schon im Jahr 1864 verkündeten „Syllabus errorum“, eine Sammlung vermeintlicher liberaler Irrtümer der Zeit, die im allgemeinen als kirchliche Kampfansage an die moderne Welt im allgemeinen und an den politischen Liberalismus im besonderen aufgefasst wurde ${ }^{28}$.

In Deutschland selbst wiederum zählt die Reichsgründung von 1871 zu den weiteren wichtigen Voraussetzungen des Kulturkampfs, denn die kleindeutsche Einigung unter Führung der norddeutsch-protestantischen Vormacht Preußen wurde von vielen traditionell "großdeutsch“ orientierten west- und süddeutschen Katholiken abgelehnt oder doch wenigstens mit Misstrauen betrachtet ${ }^{29}$. Der früher in Deutschland, vor allem im Alten Reich, dominierende Katholizismus war durch das Ausscheiden Österreichs mit einem Mal - und zwar erstmals in der neueren deutschen Geschichte - zur Minderheitenkonfession geworden ${ }^{30}$. Der offene Kampf einflussreicher, vor allem süddeutscher katholischer Kreise gegen ein kleindeutsches Reich ${ }^{31}$ hatte beim neuen Reichskanzler Bismarck den Verdacht geweckt, dass sich im katholischen Deutschland so etwas wie eine Fundamentalopposition gegen die neue politische Ordnung vorbereitete ${ }^{32}$, und die böse Vermutung, hier seien klerikale Dunkelmänner und „Reichsfeinde“ am Werk, wurde von den in dieser Zeit politisch sehr einflussreichen und mit der Regierung Bismarck eng zusammenarbeitenden Liberalen noch bewusst verstärkt ${ }^{33}$.

28 Vgl. Nipperdey, T.: Deutsche Geschichte 1866-1918, Bd. 2, S. 365 ff.; Friedrich, M.: Kirche im gesellschaftlichen Umbruch, S. 108 ff.; zur politischen Bedeutung des „Syllabus“ siehe auch die Bemerkungen bei Huber, E. R.: Deutsche Verfassungsgeschichte, Bd. 4, S. 652-654.

29 Zu diesem Thema siehe allgemein Ritter, Gerhard: Großdeutsch und kleindeutsch im 19. Jahrhundert. In: Derselbe: Lebendige Vergangenheit, Beiträge zur historisch-politischen Selbstbesinnung. München 1958, S. 101-125; Wandruszka, Adam: Großdeutsche und kleindeutsche Ideologie. In: Deutschland und Österreich. Hrsg. v. R. A. Kann; F. Prinz. Wien - München 1980, S. 110-142.

30 Hierzu vor allem Nowak, K.: Geschichte des Christentum, S. 149 ff.

31 Hierbei tat sich zwischen 1868 und 1871 vor allem die bayerische „Patriotenpartei“ hervor; vgl. Schieder, Theodor: Die kleindeutsche Partei in Bayern in den Kämpfen um die nationale Einheit. (Münchener historische Abhandlungen I, 12). München 1936, S. 193 ff., 237 ff.; Hartmannsgruber, Friedrich: Die bayerische Patriotenpartei 1868-1887. (Schriftenreihe zur bayerischen Landesgeschichte, 82). München 1986, S. 362 ff.

32 Zur Einschätzung Bismarcks siehe neben Nipperdey, T.: Deutsche Geschichte 1866-1918. Bd. 2. S. 371 ff. Auch Pflanze, Otto: Bismarck. Bd. 1. Der Reichsgründer. München 1997, S. 691 ff.; aus der älteren Literatur vor allem Bornkamm, Heinrich: Die Staatsidee im Kulturkampf. (1950), Neudruck Darmstadt 1969, bes. S. $41 \mathrm{ff}$.

33 Vgl. Sheehan, James J.: Der deutsche Liberalismus - Von den Anfängen im 18. Jahrhundert bis zum Ersten Weltkrieg 1770-1914. München 1983, S. 162 ff. 
Otto von Bismarck selbst war in dieser Zeit vor allem mit den Problemen der inneren Einigung Deutschlands befasst. Nach offizieller Auffassung war das neue Reich als ein Bund aller deutschen Fürsten gegründet worden; es handelte sich dabei zwar um einen Bundesstaat mit starken föderalen Elementen ${ }^{34}$. Nicht der Deutsche Kaiser allein, sondern alle vereinigten Bundesfürsten zusammen galten offiziell als Inhaber der Souveränität im Deutschen Reich. Das politische Organ der Fürsten und der einzelstaatlichen Regierungen auf gesamtdeutscher Ebene war der Bundesrat, der beim Gesetzgebungsverfahren eine sehr bedeutende Rolle spielte ${ }^{35}$. Angesichts dieser inneren Konstellation versuchte Bismarck in den ersten Jahren nach der Reichsgründung vor allem die unitarischen Elemente im neuen Reich zu stärken, und dies wiederum kam dem liberalen Ziel einer inneren politischen Modernisierung in Deutschland in starkem Maße entgegen.

$\mathrm{Zu}$ den Reformen in einem unitarischen Sinne - mit der Absicht einer Unterstützung aller den inneren Zusammenhalt im neuen Reich stärkenden Elementen - sollte nach Bismarcks Vorstellung vor allem eine Zurückdrängung der politischen wie auch der gesellschaftlichen und institutionellen Position der Kirchen gehören ${ }^{36}$; dies deckte sich weitgehend mit zentralen Forderungen der Liberalen. Konkret bedeutete dies etwa eine deutliche Reduzierung, wenn nicht sogar die Abschaffung der kirchlichen Einflussnahme auf die schulische und die universitäre Bildung, etwa die Abschaffung oder wenigstens Marginalisierung der konfessionellen Schulen. Und ebenfalls sollte eine alte, seit Jahrzehnten erhobene Forderung der Liberalen umgesetzt werden, die mit Bismarcks Ziel übereinstimmte: die Einführung der Zivilehe.

Der Grundzug dieser und anderer Reformen, die der deutsche Kanzler schon bald nach der Reichsgründung in Angriff nahm, war im Kern darauf gerichtet, eine stärkere politische wie gesellschaftliche Integration der Deutschen in den neuen Staat zu erreichen, und zwar durch Vereinheitlichung und durch staatliche Übernahme bestimmter Funktionen, die bis dahin von der Kirche ausgeübt worden waren. Mit der Zurückdrängung des kirchlichen Einflusses beabsichtigte man nicht nur den Staat und dessen Befugnisse zu stärken, sondern auch die sich immer noch vernehmbar artikulierenden konfessionellen Differenzen in ihrer Bedeutung, soweit es möglich schien, zu reduzieren. Die Absicht einer eigentlichen Trennung von Kirche und Staat, die Bismarck damals von seinen Gegnern katholischer wie auch evangelischer Konfession unterstellt wurde ${ }^{37}$, wurde von ihm jedoch niemals vertreten. Wohl aber sollte der immer noch große Einfluss der Konfessionen und

34 Dazu neuerdings Grimm, Dieter: War das Deutsche Kaiserreich ein souveräner Staat? In: Das Deutsche Kaiserreich in der Kontroverse. Hrsg. v. S.O. Müller; C. Torp. Göttingen 2009, S. 86-101, und demnächst Kraus, Hans-Christof: Das Deutsche Kaiserreich als monarchischer Bundesstaat (im Druck; erscheint 2013).

35 Grundlegend zur Reichsverfassung die detaillierte Gesamtdarstellung und Deutung von Huber, E. R.: Deutsche Verfassungsgeschichte, Bd. 3, passim.

36 Diesen Aspekt hebt - im Anschluss an Bornkamm, H.: Die Staatsidee im Kulturkampf, S. 41 ff. - stark hervor Thadden, Rudolf von: Bismarck - Ein Lutheraner? In: Derselbe: Weltliche Kirchengeschichte. Göttingen 1989, S. 146-163, bes. S. $151 \mathrm{ff}$.

37 Der im Jahr 1872 aus Protest gegen Bismarcks Kulturkampfpolitik zurückgetretene preußische Kultusminister Heinrich von Mühler hat genau diesen Vorwurf erhoben; vgl. Reichle, Walter: Zwischen Staat und Kirche - Das Leben und Wirken des preußischen Kultusministers Heinrich v. Mühler, dargestellt unter Benutzung des schriftlichen Nachlasses des Ministers. Berlin 1938, S. 328 f. 
Kirchen im damaligen Deutschland durch staatliche Eingriffe deutlich reduziert und auf diese Weise eine möglichst umfassende Neuregelung des Verhältnisses zwischen Staat und Kirche erreicht werden: nicht Trennung beider Bereiche, wohl aber „friedliche Grenzregelung statt unklarer Rechtsüberschneidungen und dauernder Reibungsmöglichkeiten“ und auf diese Weise „Scheidung der staatlichen und kirchlichen Gewalt“"38.

Es ist bekannt, dass alle diese Maßnahmen zu einem schweren Konflikt zwischen dem Staat und der katholischen Kirche führten, der am Ende nur mühsam und nicht ohne Gesichtsverlust für beide Seiten wieder beigelegt werden konnte. Bismarck, der im allgemeinen ein sehr erfahrener politischer Taktiker war und der die Stärke der gegnerischen Kräfte in aller Regel richtig einzuschätzen verstand, beging dieses Mal wenigstens mit einem Teil seiner Maßnahmen einen schweren Fehlgriff. Nicht nur unterschätzte er entschieden den Widerstandsgeist und die Regenerationskraft des deutschen Katholizismus, sondern er schätzte auch die protestantischen Gegenkräfte falsch ein, denn innerhalb der evangelischen Kirche, vor allem auch in konservativ-protestantischen Kreisen, begannen sich bald Kritik und Widerstand gegen bestimmte säkularisierende Maßnahmen der Regierung Bismarck bemerkbar zu machen ${ }^{39}$. Man darf in diesem Zusammenhang die bedeutsame Tatsache nicht unterschätzen, dass im norddeutschen Protestantismus immer noch ein konfessionelles Luthertum dominierte, „das sich als Gegenbewegung zum Rationalismus des preußischen Unionskirchentums entwickelt hatte und in zentralen Vorstellungen dem Katholizismus näher stand als der Welt des liberalen Protestantismus" 40 .

Die sich bald im konservativen Protestantismus (wenn auch zuerst nur vorsichtig) artikulierenden Widerstände gegen die neupreußische Kirchenpolitik erschienen dem Kanzler, der in Personalunion zugleich das Amt des preußischen Ministerpräsidenten innehatte, insofern besonders fatal, als er sich in seiner Regierung bisher - seit seinem Amtsantritt im Jahr 1862 - auf die Konservative Partei, der er selbst entstammte, unbedingt hatte verlassen können ${ }^{41}$. Dass sich seit Beginn der 1870er Jahre und den jetzt einsetzenden Kulturkampfmaßnahmen der Regierung wenigstens ein Teil der streng christlich-kirchlich orientierten protestantischen Konservativen von Bismarck abwandte, seine Politik nicht nur nicht mehr unterstützte, sondern z. T. sogar öffentlich kritisierte $^{42}$, konnte in der Konsequenz die Stellung Bismarcks, wenigstens im Königreich PreuBen, erheblich schwächen. Denn jetzt hatte er nicht nur die katholische Zentrumspartei gegen sich, sondern auch noch einen Teil der protestantischen Konservativen, während er sich auf der anderen Seite nur noch auf die liberalen Parteien verlassen konnte, denen wiederum an einer weiteren Verschärfung und Zuspitzung der Auseinandersetzung

38 So treffend Bornkamm, H.: Die Staatsidee im Kulturkampf, S. 43 f.

39 Dazu im einzelnen: Schulte, Engelbert: Die Stellung der Konservativen zum Kulturkampf 1870-1878. Phil. Diss. Köln 1959; Bornkamm, H.: Die Staatsidee im Kulturkampf, S. 24 ff.; Hilge, Friedrich: Die Evangelische Kirche und der Kulturkampf (unter besonderer Berücksichtigung Preußens). Diss. phil. Osnabrück 1999.

40 Thadden, R. von: Bismarck - ein Lutheraner, S. 149.

41 Vgl. dazu Kraus, Hans-Christof: Bismarck und die preußischen Konservativen (Friedrichsruher Beiträge, Bd. 12). Friedrichsruh 2000. 
mit der katholischen Kirche keineswegs ungelegen war. Die Konservative Partei wurde auf diese Weise plötzlich zu einem Unsicherheitsfaktor der Bismarckschen Politik, und auch diese Tatsache hat zum Abbruch des Kulturkampfes gegen Ende der 1870er Jahre wenigstens mit beigetragen.

Man darf in diesem Zusammenhang keineswegs vergessen, dass sich zentrale Aspekte der Bismarckschen Kirchenpolitik in gleicher Weise gegen die bisherigen Rechte und Befugnisse beider Kirchen und beider großen Konfessionen richtete, denn die Kontrolle über die jeweiligen konfessionell geprägten Schulen übten beide Kirchen in gleicher Weise aus, und durch eine weitere Maßnahme, die Einführung einer rechtlich verpflichtenden Ziviltrauung in Deutschland, sollte den Kirchen darüber hinaus noch ein anderes zentrales Recht genommen werden, das ihnen bisher verblieben war. Die säkularisierenden Tendenzen und die in ihrer Konsequenz wenigstens absehbaren Folgen dieser Kulturkampfpolitik wurden von vielen Gläubigen beider Konfessionen und parteipolitisch besonders von dem streng christlich orientierten rechten Flügel der Konservativen konsequent abgelehnt ${ }^{43}$.

Worin bestanden nun die wichtigsten Einzelmaßnahmen des von Bismarck seit 1871 eingeleiteten Kulturkampfes? Der Verfassungshistoriker Ernst Rudolf Huber hat diese staatlichen Maßregeln in zwei deutlich zu unterscheidende Gruppen eingeteilt: Er unterscheidet zum einen die Repressionsgesetze, und zum anderen die Strukturgesetze ${ }^{44}$. Zu den Repressionsgesetzen gehörten alle diejenigen Maßnahmen, die auf die Unterdrückung und Einschränkung kirchlicher Handlungen und Einrichtungen gerichtet waren, d. h. alle sich direkt gegen die Kirche und deren Funktionsträger richtenden Kampfmaßnahmen, - und zu den Strukturgesetzen sind alle diejenigen staatlichen Maßnahmen zu zählen, „die darauf angelegt waren, bestimmte Einrichtungen des staatlichen oder kirchlichen Lebens oder bestimmte zwischen Staat und Kirche bestehende Beziehungen strukturell und auf Dauer neu zu ordnen. Ihr Ziel war ... die Verwirklichung einer neuen Rechtskonzeption"45, vorgenommen in unverkennbar säkularisierender Absicht. Alle diese Gesetze waren teils Landesgesetze (in diesem Fall vor allem auf den größten deutschen Bundesstaat, das Königreich Preußen bezogen), teils aber auch Reichsgesetze (also mit Gültigkeit für ganz Deutschland) ${ }^{46}$.

$\mathrm{Zu}$ den Repressionsgesetzen gehörten zunächst einmal der schon im Dezember 1871 verabschiedete sog. „Kanzelparagraph“, der denjenigen Geistlichen, die im Rahmen ihrer Predigten staatliche Institutionen und Maßnahmen scharf angriffen und kritisierten, Gefängnishaft androhte. Hinzu kamen in den folgenden Jahren weitere Maßnahmen:

43 Vgl. Reichle, W.: Zwischen Staat und Kirche, S. 443 ff.; Schulte, E.: Die Stellung der Konservativen zum Kulturkampf, S. 84 ff. u. a.; Kraus, Hans-Christof: Ernst Ludwig von Gerlach. Politisches Denken und Handeln eines preußischen Altkonservativen (Schriftenreihe der Historischen Kommission bei der Bayerischen Akademie der Wissenschaften, 53), Bde. 1-2, Göttingen 1994, hier Bd. 2, S. 868 ff.

Vgl. Huber, E. R.: Deutsche Verfassungsgeschichte, Bd. 4, S. 694 ff.

46 Einen guten Überblick über die einzelnen Kulturkampfgesetze, deren Inhalte, Bedeutung und Problematik liefert neben Schmidt-Volkmar, E.: Der Kulturkampf, S. 60-146, vor allem Huber, E. R.: Deutsche Verfassungsgeschichte, Bd. 4, S. 697-745. 
einmal das im Juli 1872 beschlossene Jesuitengesetz, das ein Verbot des Jesuitenordens für das gesamte Reichsgebiet verfügte, sodann das Expatriierungsgesetz vom Mai 1874, das unter bestimmten Bedingungen katholischen Geistlichen, die gegen geltendes Recht verstoßen hatten, die Staatsbürgerschaft aberkennen und anschließend die Ausweisung aus Deutschland verfügen konnte, endlich das Sperrgesetz vom April 1875, mit dessen Hilfe bestimmten geistlichen Funktionsträgern oder kirchlichen Einrichtungen die staatliche finanzielle Unterstützung entzogen werden konnte. Am Ende stand das ebenfalls im Frühjahr 1875 verabschiedete Gesetz über Orden und Kongregationen, durch dessen Bestimmungen katholische Orden in bestimmten Fällen verboten werden konnten ${ }^{47}$.

Diesen Repressionsgesetzen wiederum, von denen die meisten später, nach dem Ende des Kulturkampfes wieder aufgehoben wurden $^{48}$, standen die Strukturgesetze gegenüber, die das Verhältnis zwischen Staat und Kirche im Deutschen Reich dauerhaft reformieren und verändern sollten. Als erstes dieser zweiten Sorte von gesetzlichen Maßnahmen muss das (preußische) Schulaufsichtsgesetz vom März 1872 gelten, durch dessen Regelungen alle öffentlichen und privaten, auch die kirchlichen Schulen der alleinigen staatlichen Aufsicht unterstellt wurden; auch die Leitung des Unterrichts wurde nun - mit der einzigen Ausnahme des Religionsunterrichts an den Volksschulen - vom Staat übernommen.

$\mathrm{Zu}$ den Strukturgesetzen gehörten ebenfalls die sog. Maigesetze des Jahres 1873, also das „Gesetz über die Vorbildung und Anstellung der Geistlichen“, das eine wissenschaftliche Staatsprüfung für alle katholischen Geistlichen vorsah, sodann das „Gesetz über die kirchliche Disziplinargewalt und die Errichtung des Gerichtshofs für kirchliche Angelegenheiten“, das den Einfluss der römischen Kurie auf die inneren Verhältnisse der deutschen Kirche stark einschränkte; es folgte ein Gesetz zur Einschränkung des Gebrauchs kirchlicher Straf- und Zuchtmittel und endlich das „Gesetz betreffend den Austritt aus der Kirche“, das für alle Kirchen und Konfessionen rechtliche und verwaltungstechnische Rahmenbedingungen für den Kirchenaustritt festlegte.

In den Jahren 1874 und 1875 folgten noch zwei weitere Gesetze, denen besonders starke säkularisierende Wirkungen zugeschrieben werden konnten: das Gesetz über die Einführung der obligatorischen Zivilehe in Preußen vom März 1874, und ein Jahr später, im Februar 1875, das Reichszivilehegesetz, durch das die im Jahr zuvor zuerst nur in Preußen eingeführte Zivilehe auf das gesamte Reichsgebiet ausgedehnt wurde ${ }^{49}$. Neugeordnet wurde im Rahmen dieser Gesetze ebenfalls das gesamte Personenstandswesen ${ }^{50}$, das in Deutschland nun reichsweit vereinheitlicht und verstaatlicht wurde. Diese Maß-

47 Die wichtigsten Gesetzestexte und wichtige weitere Quellen zum Kulturkampf finden sich in: Staat und Kirche im 19. und 20. Jahrhundert. Dokumente zur Geschichte des deutschen Staatskirchenrechts. Hrsg. E. R. Huber; W. Huber. Bd. II. Berlin 1976, S. 395-928.

48 Zur Beilegung des Kulturkampfes siehe unten, Abschnitt IV.

49 Abdruck in: Staat und Kirche im 19. und 20. Jahrhundert, Bd. 2, S. 631 (Nr. 297). Ein erster Versuch zur Einführung der Zivilehe und einer amtlichen „Beurkundung des Personenstandes“ war 1871 noch gescheitert, siehe dazu die Dokumentation bei Constabel, Adelheid (Bearb.): Die Vorgeschichte des Kulturkampfes. Quellenveröffentlichung aus dem Deutschen Zentralarchiv. Berlin (Ost) 1956, S. $144 \mathrm{ff}$.

50 Vgl. Huber, E. R.: Deutsche Verfassungsgeschichte, Bd. 4, S. 733 f.; Nipperdey, T.: Deutsche Geschichte 18661918, Bd. 2, S. 375. 
nahmen waren auf Dauer hin angelegt, und sie gelten im Kern ihrer Bestimmungen bekanntlich bis heute; man wird in ihnen einen wichtigen Schritt auf dem Weg zur Integration, zur Vereinheitlichung und zur inneren Festigung des 1871 neu begründeten deutschen Nationalstaats sehen können - und als solcher war er von Bismarck auch beabsichtigt.

\section{III.}

Die Entwicklung und der Ausgang des Kulturkampfs im Deutschland der 1870er Jahre ist entscheidend mitbestimmt worden durch den erstaunlich starken, ausgeprägten und zähen Widerstand der katholischen Kirche im Allgemeinen sowie des von Bismarck in seiner Bedeutung unterschätzten politischen Katholizismus, also der Deutschen Zentrumspartei, im Besonderen ${ }^{51}$. Die Heftigkeit des Widerstands, der sich vor allem gegen die Repressionsgesetze richtete, trug wesentlich mit dazu bei, dass sich Bismarck gegen Ende der 1870er Jahre zum Abbruch des Kulturkampfs und zu dessen Beilegung, die sich allerdings eine Reihe von Jahren lang hinziehen sollte, mehr oder weniger gezwungen sah. Und hierzu trugen nicht zuletzt ebenfalls deutlich veränderte politische Rahmenbedingungen im Deutschen Reich mit bei.

Schon seit 1872 erhob sich im katholischen Deutschland ein entschlossener, vom Papst und der römischen Kurie massiv unterstützter Widerstand gegen die Kulturkampfgesetze, der im öffentlichen Streit um die Maigesetze von 1873 seinen ersten Höhepunkt fand. Am Ende stellte sich in den Jahren zwischen 1872 und 1879 heraus, dass Bismarck in einem Punkt gerade das Gegenteil von demjenigen erreichte, was er eigentlich beabsichtigt hatte. Katholische Vereine, katholische Presse und Verlage erlebten einen ebenso deutlichen Aufschwung wie andere Bereiche der katholischen Laienbewegung; der preußische und deutsche Katholizismus ging nicht geschwächt, sondern tatsächlich innerlich und äußerlich gestärkt aus dem Kulturkampf hervor ${ }^{52}$.

Mit einiger Verzögerung schaltete sich seit dem Februar 1875 auch Papst Pius IX. öffentlich in die Auseinandersetzung seiner Kirche mit dem preußischen Staat und dem Deutschen Reich ein. Er kritisierte in einem Schreiben an die preußischen Bischöfe ${ }^{53}$ die bisher im Rahmen der Kulturkampfmaßnahmen erlassenen Kirchengesetze sowie ebenfalls die Absetzung und Inhaftierung mehrerer preußischer Bischöfe durch den Staat; alle diese Maßnahmen, einschließlich der Gesetze, wurden vom katholischen Kirchenoberhaupt für ungültig erklärt. In der Tat war diese Ungültigkeitserklärung, wie

51 Ausführlich hierzu: Bachem, Karl: Vorgeschichte, Geschichte und Politik der deutschen Zentrumspartei. Bd. 3, Köln 1927, S. 193-353; Anderson, Margaret Lavinia: Windthorst. Zentrumspolitiker und Gegenspieler Bismarcks (Forschungen und Quellen zur Zeitgeschichte 14). Düsseldorf 1988, S. 166-204.

52 Vgl. die Hinweise bei Morsey, R.: Der Kulturkampf, S. 92 f.. Sowie Ross, Ronald J.: The Failure of Bismarck's Kulturkampf. Catholicism and State Power in Imperial Germany, 1871-1887. Washington D. C. 1998, bes. S. 53 ff. u. a.

53 Abdruck in: Staat und Kirche im 19. und 20. Jahrhundert, Bd. 2, S. 651-655 (Nr. 307); dazu auch Huber, E. R.: Deutsche Verfassungsgeschichte, Bd. 4, S. 732 f.; Franz, G.: Kulturkampf, S. 238. 
mit Recht bemerkt worden ist, „der schwerste Gegenangriff, den die Kurie während der langen Auseinandersetzungen unternahm“54. Denn in dieser Erklärung war zugleich der bindende Befehl an alle Katholiken enthalten, sich der Teilnahme am Vollzug aller gegen die Kirche gerichteten staatlichen Maßnahmen zu enthalten, und zwar bei Androhung der Strafe der Exkommunikation ${ }^{55}$.

Aber nicht nur in den mehrheitlich katholischen Landesteilen Preußens und einiger anderer deutscher Bundesstaaten verstärkte sich mit der zunehmenden Verschärfung der staatlichen Maßnahmen der Widerstand, sondern auch innerhalb der Konservativen Partei und in christlich-konservativen Kreisen, darunter nicht zuletzt am königlichen und kaiserlichen Hof in Berlin, artikulierten sich immer deutlichere Bedenken gegen den harten kirchenpolitischen Konfrontationskurs der Regierung Bismarck ${ }^{56}$. Dies hing vor allem damit zusammen, dass gleichzeitig mit den Maßnahmen zur Zurückdrängung des Einflusses der katholischen Kirche ebenfalls eine Reform der evangelischen Kirchenverfassung vorbereitet wurde, die auf eine Stärkung des synodalen Elements sowie im Gegenzug auf eine Verminderung des traditionell monarchisch-landesherrlich orientierten Konsistorialverfassung hinauslief ${ }^{57}$. Und natürlich war die evangelische Kirche von den Strukturgesetzen unter den Kulturkampfmaßnahmen der Regierung - etwa von den Bestimmungen des Schulaufsichtsgesetzes und der Einführung der Zivilehe - ebenfalls stark betroffen. Den Säkularisierungs- und Modernisierungsschub, den diese Regelungen im Kern bedeuteten, konnten konservative Protestanten ebenso wenig akzeptieren wie die römische Kurie und die Mehrheit der deutschen Katholiken.

Schon in den Anfangsjahren des Kulturkampfes kam es daher im Zeichen des gemeinsamen Protestes zu einer vorsichtigen Annäherung zwischen Teilen der Konservativen Partei und der katholischen Zentrumspartei, und gerade diese Entwicklung wurde von Bismarck als besonders gefährlich angesehen. Er unternahm daher alles, um auf der politischen und parlamentarischen Ebene die wechselseitig traditionell vorhandenen konfessionellen Vorurteile zu stärken und den Gegensatz zwischen Katholiken und Protestanten erneut zu schüren ${ }^{58}$. Das Ziel dieser Aktionen bestand zum einen darin, die Gegensätze zwischen Konservativen und Zentrum erneut zu verschärfen; zum anderen aber bezweckte Bismarck die Neugründung einer strikt regierungstreuen Konservativen Partei. Das erste gelang zumindest partiell, das zweite allerdings vollständig: Die zerstrittene alte Konservative Partei zerfiel, und im Jahr 1876 wurde unter diskreter Regie Bismarcks die neue Deutschkonservative Partei begründet, die fortan fest auf der Seite des Kanzlers stand und dessen Politik uneingeschränkt unterstützte ${ }^{59}$.

54 Huber, E. R.: Deutsche Verfassungsgeschichte, Bd. 4, S. 733.

55 Vgl. die entsprechende Passage in Papst Pius IX. Enzyklika an den preußischen Episkopat vom 5. 2. 1875, in: Staat und Kirche im 19. und 20. Jahrhundert, Bd. 2, S. 653.

56 Vgl. Schulte, E.: Die Stellung der Konservativen zum Kulturkampf, S. 68 ff.

57 Dazu u. a. die Darstellung bei Foerster, Erich: Adalbert Falk. Sein Leben und Wirken als preußischer Kultusminister. Gotha 1927, S. 174 ff.; siehe ebenfalls Thadden, R. von: Bismarck - ein Lutheraner?, S. 150 ff. Vgl. Schulte, E.: Die Stellung der Konservativen zum Kulturkampf, S. 96 ff.; zum Zusammenhang siehe auch Ritter, Gerhard: Die preußischen Konservativen und Bismarcks deutsche Politik 1858 bis 1876. Heidelberg 1913. 
Auch die retardierende Rolle des Hofes, vor allem die Bedenken des alten, im Jahr 1875 bereits achtzigjährigen Königs und Kaisers, hatte Bismarck zu Anfang vermutlich unterschätzt. Kaiser Wilhelm I. war mit einer Reihe von Kulturkampfmaßnahmen ausdrücklich nicht einverstanden; bereits die in den 1870er Jahren zeitweilig besonders enge Zusammenarbeit Bismarcks mit den Liberalen, die den Kulturkampf mit aller Schärfe führten, war ihm ausgesprochen unangenehm. Der Amtsverzicht des christlich-konservativen Kultusministers Heinrich von Mühler, der nach seinen Auseinandersetzungen mit Bismarck die Kulturkampfpolitik nicht mehr mittragen konnte und wollte, wurde vom Kaiser äußerst ungern gesehen, und der alte Monarch versuchte ebenfalls, sich dem Schulaufsichtsgesetz und der Einführung der Zivilehe zu widersetzen ${ }^{60}$. Doch Bismarck setzte sich hier gegen Wilhelm ebenso durch wie mit seiner Berufung des neuen, stark anti-ultramontan orientierten Kulturministers Adalbert Falk als Nachfolger Mühlers ${ }^{61}$.

Insgeheim mag der alte Monarch die Befürchtungen Mühlers geteilt haben, Bismarcks eigentliches Ziel liege darin, „die Kirche und die religiösen Ideen ganz aus dem öffentlichen Leben [zu] verbannen und zu einer bloßen Privatsache zu machen“, damit also insgesamt eine „Trennung der Kirche vom Staat" ${ }^{\text {“2 }}$ vorzubereiten. Und es ist ebenfalls bekannt, dass er seine Stellung und seine Aufgaben als Summus episcopus der evangelischen Landeskirche in Preußen stets sehr ernst nahm und deshalb den Überlegungen und Plänen der Regierung für eine grundlegende Reform der evangelischen Kirchenverfassung und die „Einführung synodaler Strukturelemente in die bislang kaum gewandelte Pastoren- und Behördenkirche“ ausgesprochen kritisch gegenüberstand; auch in dieser Hinsicht hatte Bismarck Rücksicht auf den König zu nehmen ${ }^{63}$.

Zusammenfassend lässt sich feststellen: Alle diese Widerstände gegen die Kulturkampfmaßnahmen im protestantischen und konservativen Lager und ebenfalls die immer stärker werdenden Bedenken des Trägers der Krone konnte Bismarck infolge seiner exorbitant starken Stellung und seiner politischen Doppelfunktion als Reichskanzler und preußischer Ministerpräsident ${ }^{64}$ zwar eine Zeitlang außer Acht lassen - jedoch nicht auf Dauer ignorieren. Auch dieser Aspekt hat mit dazu beigetragen, dass der Kulturkampf gegen Ende der 1870er Jahre abgebrochen und zu einem für beide Seiten halbwegs akzeptablen Ende gebracht werden konnte.

1876-1918. Boston 1988, S. 13-22.

60 Vgl. Marcks, Erich: Kaiser Wilhelm I. Hrsg. von K. Pagel. Berlin 1943 ${ }^{9}$, S. 311.

61 Vgl. Ruhenstroth-Bauer, Renate: Bismarck und Falk im Kulturkampf. Heidelberg 1944.

62 Reichle, W.: Zwischen Staat und Kirche, S. 328.

63 Hierzu vgl. die Bemerkungen bei Thadden, R. von: Bismarck - ein Lutheraner, S. 151 ff.; das Zitat S. 152 f.

64 Nur während etwa eines Jahres (Dezember 1872 bis November 1873) amtierte der bisherige Kriegsminister Albrecht von Roon für kurze Zeit als preußischer Ministerpräsident; anschließend übernahm Bismarck dieses Amt erneut selbst. 


\section{IV.}

Der Anfang vom Ende des Kulturkampfs ist auf das Jahr $1878 \mathrm{zu}$ datieren. Zwei Ereignisse markieren diesen historischen Einschnitt: erstens die von Bismarck in diesem Jahr angebahnte und vollzogene innenpolitische Wende im Zeichen des Antiliberalismus und einer damit einhergehenden neuen Hinwendung zu den Konservativen und zur Zentrumspartei ${ }^{65}$; diese Wende war wesentlich mit bestimmt worden durch die wenige Jahre zuvor ausgebrochene internationale Wirtschaftskrise, die im Deutschen Reich einen Wechsel vom Freihandel zum Schutzzollsystem erforderte; dieser Wechsel widersprach den liberalen Glaubenssätzen einer freien Wirtschaft derart fundamental, dass ihn ein Teil der liberalen Kräfte, auf die sich Bismarck seit Beginn der 1870er Jahre gestützt hatte, nicht mehr mittragen konnte und wollte.

Das andere Ereignis, in dem sich das Ende der Auseinandersetzung ankündigte, war der Tod Papst Pius IX. und die Wahl seines versöhnungsbereiten Nachfolgers Leo XIII. im Februar 1878. Nicht nur die preußische Regierung und Bismarck als Reichskanzler waren an einem Ende des Kulturkampfs interessiert, sondern auch die Kurie selbst; dem neuen Papst scheint klar gewesen zu sein, dass eine weitere Verschärfung des Konflikts in Deutschland der Kirche langfristig nur schaden konnte, nicht zuletzt angesichts weiterer Auseinandersetzungen in Österreich, Frankreich und vor allem in Italien ${ }^{66}$. Die in der nun folgenden Zeit also von beiden Seiten betriebene Beilegung des Kulturkampfes zog sich noch eine Reihe von Jahren hin; jetzt wurden vor allem die von der Regierung Bismarck seit 1872 erlassenen Repressionsgesetze nach und nach vollständig abgebaut ${ }^{67}$.

Beide Seiten zeigten am Ende Kompromissbereitschaft und waren innerhalb gewisser Grenzen tatsächlich zum Nachgeben bereit; mit den sog. „Milderungsgesetzen“ und den „Friedensgesetzen“ der 1880er Jahre wurde schließlich ein für beide Seiten tragbarer und akzeptabler Kompromiss erreicht. Die durch die staatlichen Absetzungen bisher vakanten Bischofsstühle wurden wiederbesetzt, und auch die im Jahr 1872 abgebrochenen diplomatischen Beziehungen zwischen dem preußischen Staat und dem Heiligen Stuhl wurden 1882 erneut aufgenommen. Gleichwohl darf nicht übersehen werden, dass die politischen und auch die psychologischen Nachwirkungen des Kulturkampfes in Deutschland jahrzehntelang zu verspüren waren; der Gegensatz zwischen den Konfessionen hatte sich in den 1870er Jahren deutlich verschärft und konnte nur langsam gemildert und abgebaut werden.

65 Hierzu ausführlich Nipperdey, T.: Deutsche Geschichte 1866-1918, Bd. 2, S. 382-408; Pflanze, Otto: Bismarck. Bd. 2. Der Reichskanzler. München 1998, S. 237-261; Sheehan, J. J.: Der deutsche Liberalismus, S. 214-223.

66 Aufschlussreich hierzu neuerdings: Borutta, Manuel: Antikatholizismus. Deutschland und Italien im Zeitalter der europäischen Kulturkämpfe (Bürgertum NF 7). Göttingen 2010; siehe ebenfalls die Beiträge in: Kulturkampf in Europa im 19. Jahrhundert. Hrsg. Ch. Clark; W. Kaiser. Leipzig 2003.

67 Vgl. den fundierten Überblick bei Morsey, R.: Der Kulturkampf, S. 93 ff., sowie die sehr detaillierte Darstellung bei Huber, E. R.: Deutsche Verfassungsgeschichte, Bd. 4, S. 767-804; ebenfalls Martin, Matthias: Der katholische Weg ins Reich. Der Weg des deutschen Katholizismus vom Kulturkampf hin zur staatstragenden Kraft. Frankfurt a. M. 1998, S. 156 ff. 
Ebenfalls darf nicht vergessen werden, dass die von Bismarck seit 1872 durchgesetzten Strukturgesetze, die das Verhältnis zwischen Staat und Kirche in Deutschland in mehreren Bereichen völlig neu geregelt hatten, auch weiterhin in Kraft blieben. Hier gab es keinen Rückgang auf ältere Regelungen; die Zivilehe oder die staatliche Schulaufsicht blieben weiter bestehen, und dieses Faktum hatte nicht nur die katholische, sondern auch die evangelische Kirche zu akzeptieren. Nicht zuletzt konnte Bismarck im Jahr 1873 eine neue, etwas liberalere evangelische Kirchenverfassung, die synodale und konsistoriale Elemente miteinander kombinierte, gegen heftige Widerstände der Konservativen durchsetzen ${ }^{68}$. Den beabsichtigten Säkularisierungsschub in Deutschland, der in der Zurückdrängung der Kirche aus einigen ihrer früher wahrgenommenen gesellschaftlichen, bildungspolitischen und kulturellen Funktionen bestand, hatte Bismarck in der Tat durchsetzen können, aber der Preis war sehr viel höher gewesen, als er es vor Beginn des Konflikts vermutlich geahnt und erwartet hatte.

Fragt man abschließend nach den wichtigsten Motiven, die Bismarck ab 1878 zu einer Beilegung des Kulturkampfs und zu einer Einigung mit der katholischen Kirche veranlassten, so sind in erster Linie vier zentrale Aspekte zu nennen:

1. Der unerwartet starke Widerstand des größeren Teils der deutschen Katholiken, auch und gerade in den katholischen Gebieten Preußens, rief im gesamten Deutschen Reich in zunehmendem Maße innere Unruhe und Unzufriedenheit auch mit den bestehenden politischen Verhältnissen hervor. Nicht zuletzt verschärfte sich wenigstens partiell der Gegensatz zwischen den Konfessionen und auch zwischen den politischen Parteien; die katholische Zentrumspartei drohte in einen Zustand parlamentarischer Fundamentalopposition gegen den neuen Staat zu geraten - und eben dies wollte Bismarck vermeiden.

2. Auch auf der evangelisch-konservativen Seite artikulierte sich ein ungewöhnlich deutlicher Widerstand gegen die Kirchenpolitik der Regierung Bismarck sowie mindestens gegen einen Teil der Kulturkampfgesetze, von denen die evangelische Kirche mit betroffen war. Konservative Protestanten lehnten das Schulaufsichtsgesetz und das Zivilehegesetz wegen seiner säkularisierenden, die traditionellen Rechte der Kirchen deutlich einschränkenden Wirkungen ebenfalls ab. Hinzu kam die Tatsache, dass der alte König und Kaiser Wilhelm I. den Kulturkampfmaßnahmen der preußischen Regierungen und auch den entsprechenden Regelungen auf Reichsebene zunehmend kritisch und ablehnend gegenüber stand und seinen Ministerpräsidenten und Reichskanzler Bismarck immer wieder zur Mäßigung mahnte.

3. Die Wende der Bismarckschen Politik gegen Ende der 1870er Jahre wurde durch verschiedene Ereignisse geradezu erzwungen: Hierzu gehörten außenpolitische Notwendigkeiten ebenso wie der Beginn einer schweren internationalen Wirtschaftskrise und endlich auch die insgesamt für die Entwicklung des neuen Deutschen Reiches schädlichen kirchlich-politischen Konflikte innerhalb Deutschlands selbst. Die Umsetzung bestimmter, von der Regierung durchgesetzter Einzelmaßnahmen im Rahmen des Kulturkampfs entfalteten im Inneren derart schädliche Wirkungen, dass der Kanzler gehalten war,

68 Vgl. Thadden, R. von: Bismarck - ein Lutheraner?, S. 152 ff.; Huber, E. R.: Deutsche Verfassungsgeschichte, Bd. 4, S. 849-853. 
diesen Konflikt nach Möglichkeit bald zu beenden.

4. Bismarck hatte mit den von ihm durchgesetzten Strukturgesetzen, die den Einfluss der Kirchen beider Konfessionen auf die Entwicklung der Schulen und die Gestaltung des Unterrichts verringerten, die die Eheschließung säkularisierten, die dem Staat darüber hinaus einen stärkeren Einfluss auf die Rekrutierung des geistlichen und kirchlichen Führungspersonals im Deutschen Reich sicherten, wenigstens einen Teil dessen erreicht, was er seit den ersten Kulturkampfmaßnahmen des Jahres 1871 angestrebt hatte. Der neue deutsche Nationalstaat hatte einen Modernisierungs- und Säkularisierungsschub erhalten, der nicht mehr rückgängig zu machen war und dessen Wirkungen sich erst in den folgenden Jahrzehnten umfassend entfalten sollten.

Insgesamt also wird man den Kulturkampf im den ersten Jahren nach der Gründung des Deutschen Reiches als Teilaspekt des konfliktreichen Wechselspiels zwischen staatlicher und gesellschaftlicher Modernisierung einerseits und den Beharrungskräften traditioneller Kirchlichkeit und Religiosität andererseits charakterisieren können ${ }^{69}$. Hiervon waren, wie ausdrücklich noch einmal betont werden soll, beide der großen Konfessionen betroffen, nicht nur der Katholizismus allein. Und man wird ebenfalls die Tatsache nicht übersehen können, dass Auseinandersetzungen ähnlicher Art zur gleichen Zeit auch in anderen Ländern Europas - vor allem in Italien und Frankreich - stattgefunden haben. Die Kulturkämpfe im Europa des späten 19. und frühen 20. Jahrhunderts gehören also in den weiteren Zusammenhang der Modernisierungskrisen, von denen die innere Entwicklung Europas in den Jahrzehnten vor dem Ersten Weltkrieg immer wieder betroffen worden ist.

\section{Bismarck, konzervativci a „Kulturkampf" v německé říši}

Článek se věnuje charakteristice a peripetiím tzv. kulturního boje, který vedl kancléř nového německého národního státu Otto von Bismarck proti katolické církvi a politickému katolicismu (strana Centrum). Autor objasňuje různé aspekty tohoto zápasu a věnuje se jeho aktérům, zejména církevním. Bismarck řešil vládní politiku často tak, že se snažil eliminovat skupiny, které se nehodily do koncepce monarchického autoritativního státu - to se týkalo katolíků nejen z důvodů ideových (starý odpor proti centralismu, vazba na nadnárodní papežství), ale též národnostních (polští duchovní). Bismarckův Kulturkampf, v němž šlo primárně o státní dohled nad školstvím a o obsazování míst duchovních a v podstatě o odejmutí nezávislého postavení katolických skupin, však neskončil Bismarckovým vítězstvím, ale spíše politickým kompromisem a aktivizací německého politického katolicismu. To také ostatně podporovaly některé konzervativní složky společnosti, smýšlející spíše tradicionalisticky.

69 Dieser Aspekt wird hervorgehoben von Loth, Wilfried: Bismarcks Kulturkampf. Modernisierungskrise, Machtkämpfe und Diplomatie. In: Religionspolitik in Deutschland. Von der Frühen Neuzeit bis zur Gegenwart. Martin Greschat zum 65. Geburtstag. Hrsg. A.D. Manteuffel; K. Nowak. Stuttgart - Berlin Köln 1995, S. 149-163, und Clark, Christopher: Kulturkampf und europäische Moderne. In: Religionskonflikte im Verfassungsstaat Hrsg. A. Reuter; H. G. Kippenberg. Göttingen 2010, S. 23-65. 